\title{
The continued need for animals to advance brain research
}

Citation for published version (APA):

Homberg, J. R., Adan, R. A. H., Alenina, N., Asiminas, A., Bader, M., Beckers, T., Begg, D. P., Blokland, A., Burger, M. E., van Dijk, G., Eisel, U. L. M., Elgersma, Y., Englitz, B., Fernandez-Ruiz, A., Fitzsimons, C. P., van Dam, A-M., Gass, P., Grandjean, J., Havekes, R., ... Genzel, L. (2021). The continued need for animals to advance brain research. Neuron, 109(15), 2374-2379.

https://doi.org/10.1016/j.neuron.2021.07.015

Document status and date:

Published: 04/08/2021

DOI:

10.1016/j.neuron.2021.07.015

Document Version:

Publisher's PDF, also known as Version of record

\section{Document license:}

Taverne

\section{Please check the document version of this publication:}

- A submitted manuscript is the version of the article upon submission and before peer-review. There can be important differences between the submitted version and the official published version of record.

People interested in the research are advised to contact the author for the final version of the publication, or visit the DOI to the publisher's website.

- The final author version and the galley proof are versions of the publication after peer review.

- The final published version features the final layout of the paper including the volume, issue and page numbers.

Link to publication

\footnotetext{
General rights Owners
rights.

- You may freely distribute the URL identifying the publication in the public portal. please follow below link for the End User Agreement:

www.umlib.nl/taverne-license

Take down policy

If you believe that this document breaches copyright please contact us at:

repository@maastrichtuniversity.nl

providing details and we will investigate your claim.
}

Copyright and moral rights for the publications made accessible in the public portal are retained by the authors and/or other copyright owners and it is a condition of accessing publications that users recognise and abide by the legal requirements associated with these

- Users may download and print one copy of any publication from the public portal for the purpose of private study or research.

- You may not further distribute the material or use it for any profit-making activity or commercial gain

If the publication is distributed under the terms of Article $25 \mathrm{fa}$ of the Dutch Copyright Act, indicated by the "Taverne" license above, 
The continued need for animals to advance brain research

\author{
Judith R. Homberg, ${ }^{1, *}$ Roger A.H. Adan, ${ }^{7}$ Natalia Alenina, ${ }^{15}$ Antonis Asiminas, ${ }^{23,24}$ Michael Bader, ${ }^{15}$ Tom Beckers, ${ }^{20}$ \\ Denovan P. Begg, ${ }^{38}$ Arjan Blokland, ${ }^{10}$ Marilise E. Burger, ${ }^{33}$ Gertjan van Dijk, ${ }^{6}$ Ulrich L.M. Eisel, ${ }^{6}$ Ype Elgersma, ${ }^{3}$ \\ Bernhard Englitz, ${ }^{2}$ Antonio Fernandez-Ruiz, ${ }^{35}$ Carlos P. Fitzsimons, ${ }^{5}$ Anne-Marie van Dam, ${ }^{9,40}$ Peter Gass, ${ }^{19}$ \\ Joanes Grandjean, ${ }^{1}$ Robbert Havekes, ${ }^{6}$ Marloes J.A.G. Henckens, ${ }^{1}$ Christiane Herden, ${ }^{12,13}$ Roelof A. Hut, ${ }^{6}$ \\ Wendy Jarrett, ${ }^{22}$ Kate Jeffrey, ${ }^{39}$ Daniela Jezova, ${ }^{30}$ Andries Kalsbeek, ${ }^{8,9}$ Maarten Kamermans, ${ }^{8,9}$ Martien J. Kas, ${ }^{6}$ \\ Nael Nadif Kasri, ${ }^{1}$ Amanda J. Kiliaan, ${ }^{1}$ Sharon M. Kolk, ${ }^{2}$ Aniko Korosi, ${ }^{5}$ S. Mechiel Korte, ${ }^{43}$ Tamas Kozicz, ${ }^{36}$ \\ Steven A. Kushner, ${ }^{3}$ Kirk Leech, ${ }^{31}$ Klaus-Peter Lesch, ${ }^{16,17,18}$ Heidi Lesscher, ${ }^{11}$ Paul J. Lucassen, ${ }^{5}$ Anita Luthi, ${ }^{29}$ Liya Ma, ${ }^{2}$ \\ Anne S. Mallien, ${ }^{19}$ Peter Meerlo, ${ }^{6}$ Jorge F. Mejias, ${ }^{5}$ Frank J. Meye, ${ }^{7}$ Anna S. Mitchell, ${ }^{21}$ Joram D. Mul, ${ }^{5}$
}

(Author list continued on next page)

\author{
${ }^{1}$ Radboud University Medical Center, Nijmegen, the Netherlands \\ ${ }^{2}$ Radboud University, Nijmegen, the Netherlands \\ ${ }^{3}$ Erasmus Medical Center, Rotterdam, the Netherlands \\ ${ }^{4}$ Center for Neurogenomics and Cognitive Research, Vrije Universiteit Amsterdam, Amsterdam, the Netherlands \\ ${ }^{5}$ Swammerdam Institute for Life Sciences, Faculty of Science, University of Amsterdam, Amsterdam, the Netherlands \\ ${ }^{6}$ Groningen Institute for Evolutionary Life Sciences, University of Groningen, Groningen, the Netherlands \\ ${ }^{7}$ University Medical Center Utrecht, Utrecht, the Netherlands \\ ${ }^{8}$ Netherlands Institute for Neuroscience (NIN), Amsterdam, the Netherlands \\ ${ }^{9}$ Amsterdam University Medical Center, University of Amsterdam, Amsterdam, the Netherlands \\ ${ }^{10}$ Maastricht University, Maastricht, the Netherlands \\ ${ }^{11}$ Faculty of Veterinary Medicine, Utrecht University, Utrecht, the Netherlands \\ ${ }^{12}$ Institute of Veterinary Pathology, Gießen, Gießen, Germany \\ ${ }^{13}$ Center of Mind Brain and Behavior (CMBB), Philipps-University of Marburg and Justus-Liebig-University Gießen, Marburg, Germany \\ ${ }^{14}$ Philipps-University of Marburg, Faculty of Psychology, Experimental and Biological Psychology, Behavioral Neuroscience, Marburg, \\ Germany \\ ${ }^{15}$ The Max Delbrück Center for Molecular Medicine in the Helmholtz Association (MDC), Berlin, Germany
}

(Affiliations continued on next page)

Policymakers aim to move toward animal-free alternatives for scientific research and have introduced very strict regulations for animal research. We argue that, for neuroscience research, until viable and translational alternatives become available and the value of these alternatives has been proven, the use of animals should not be compromised.

\section{Introduction}

With the implementation of the European directive to protect animals used in scientific procedures (\#2010/63) around 10 years ago, the European Union (EU) set high ambitions regarding the protection of animals for research purposes. This directive focused on the development and implementation of the "3Rs" alternatives (replacement, reduction, refinement), transparency (public information about the use of animals), and their harmonization across Europe. Included in the directive is the long-term goal to "stop animal research, as soon as alter- natives for specific animal research are available." We fully agree with this and share the long-term goal of minimizing and eventually even stopping animal research. To achieve this ambitious goal, in our opinion, we must develop validated alternatives for animal research where possible. Unfortunately, presently, there are limited non-animal model systems for most brain functions and diseases that mirror the complex structure of the real brain. Therefore, we argue that, for neuroscience research, until viable and translational alternatives become available and the value of these alternatives has been proven, the use of animals should not be compromised. Instead, we must refine and optimize animal welfare and develop animal models with high translational validity (Figure 1A).

The ethics of animal use

We begin with an acknowledgment of the difficult issues raised by animal experimentation. As compassionate beings, we recognize that caging animals and then subjecting them to procedures that may be unpleasant or even sometimes painful goes against our natural instinct to treat other creatures with kindness. Worldwide, a majority of people eat meat. In addition, many animals are 
Umberto Olcese,$^{5}$ Azahara Oliva González, ${ }^{35}$ Jocelien D.A. Olivier, ${ }^{6}$ Massimo Pasqualetti, ${ }^{26}$ Cyriel M.A. Pennartz, ${ }^{5}$ Piotr Popik, ${ }^{28}$ Jos Prickaerts, ${ }^{10}$ Liset M. de la Prida, ${ }^{27}$ Sidarta Ribeiro, ${ }^{32}$ Benno Roozendaal, ${ }^{1}$ Janine I. Rossato, ${ }^{34}$ Ali-Akbar Salari, ${ }^{37}$ Regien G. Schoemaker, ${ }^{6}$ August B. Smit, ${ }^{4}$ Louk J.M.J. Vanderschuren, ${ }^{11}$ Tomonori Takeuchi, ${ }^{25}$ Rixt van der Veen, ${ }^{5}$ Marten P. Smidt, ${ }^{5}$ Vladyslav V. Vyazovskiy, ${ }^{42}$ Maximilian Wiesmann, ${ }^{1}$ Corette J. Wierenga, ${ }^{41}$ Bella Williams, ${ }^{22}$ Ingo Willuhn, ${ }^{8,9}$ Markus Wöhr, ${ }^{13,14,20}$ Monique Wolvekamp, ${ }^{2}$ Eddy A. van der Zee, ${ }^{6}$ and Lisa Genzel ${ }^{2, *}$ ${ }^{16}$ Division of Molecular Psychiatry, Center of Mental Health, University of Würzburg, Würzburg, Germany

${ }^{17}$ Laboratory of Psychiatric Neurobiology, Institute of Molecular Medicine, Sechenov First Moscow State Medical University, Moscow, Russia ${ }^{18}$ Department of Neuropsychology and Psychiatry, School for Mental Health and Neuroscience (MHeNS), Maastricht University, Maastricht, the Netherlands

${ }^{19}$ Central Institute of Mental Health, University of Heidelberg, Mannheim Faculty, Mannheim, Germany

${ }^{20} \mathrm{KU}$ Leuven, Leuven Brain Institute and Faculty of Psychology and Educational Sciences, Leuven, Belgium

${ }^{21}$ University of Oxford, Oxford, UK

22Understanding Animal Research, London, UK

${ }^{23}$ Simons Initiative for the Developing Brain, University of Edinburgh, Edinburgh EH8 9XD, UK

${ }^{24}$ Center for Translational Neuromedicine, University of Copenhagen, 2200 Copenhagen, Denmark

${ }^{25}$ Danish Research Institute of Translational Neuroscience - DANDRITE, Nordic-EMBL Partnership for Molecular Medicine, Aarhus University, Aarhus C, Denmark

${ }^{26}$ University of Pisa, Pisa, Italy

${ }^{27}$ Instituto Cajal, Consejo Superior de Investigaciones Científicas, Madrid, Spain

${ }^{28}$ Maj Institute of Pharmacology, Polish Academy of Sciences, Kraków 31-343, Poland

${ }^{29}$ Faculty of Biology and Medicine, University of Lausanne, Lausanne, Switzerland

${ }^{30}$ Institute of Experimental Endocrinology, Biomedical Research Center, Slovak Academy of Sciences, Bratislava, Slovakia

${ }^{31}$ European Animal Research Association, London, UK

${ }^{32}$ Brain Institute, Federal University of Rio Grande do Norte, Natal, Brazil

${ }^{33}$ Federal University of Santa Maria, Santa Maria, Brazil

${ }^{34}$ Department of Physiology, Federal University of Rio Grande do Norte, Natal, Brazil

${ }^{35}$ Cornell University, Ithaca, NY, USA

${ }^{36}$ Mayo Clinic, Rochester, MN, USA

${ }^{37}$ Salari Institute of Cognitive and Behavioral Disorders (SICBD), Karaj, Alborz, Iran

${ }^{38}$ School of Psychology, UNSW Sydney, Sydney, NSW, Australia

${ }^{39}$ Institute of Behavioural Neuroscience, University College London, London WC1H OAP, UK

${ }^{40}$ Amsterdam University Medical Center, Free University, Amsterdam, the Netherlands

${ }^{41}$ Biology Department, Faculty of Science, Utrecht University, Utrecht, the Netherlands

${ }^{42}$ Sleep and Circadian Neuroscience Institute, University of Oxford, Oxford, UK

${ }^{43}$ Faculty of Science, Utrecht University, Utrecht, the Netherlands

*Correspondence: judith.homberg@radboudumc.nl (J.R.H.), I.genzel@donders.ru.nl (L.G.)

https://doi.org/10.1016/j.neuron.2021.07.015

used for economic purposes (wool, transport, etc.). This implies that a majority of people are ethically comfortable with the exploitation of animals for human benefit and suggests that the discomfort with animal experimentation may have less to do with exploitation per se and more with a mistrust of science and scientists. We suggest that given this acceptance of exploitation, animal experimentation should be considered a variation of this and thus acceptable subject to the same constraints that are accepted in food and clothing production: careful husbandry, good veterinary care, minimal-stress interventions, and humane euthanasia.

The importance of animal experiments in brain research More than $35 \%$ of the EU population suffers from brain disorders affecting the lives of individuals as well as their families and loved ones (Raggi and Leonardi, 2020). Most pervasive brain disorders are highly complex, and their etiology often remains poorly understood. Common brain disorders include psychiatric disorders, neurodevelopmental disorders, neurodegenerative disorders, sensory disorders, other neurological diseases (e.g., epilepsy, brain tumors), and acquired brain injury. These disorders can be comorbid, making the combined suffering and burden for society immense.

Neuroscience is the key scientific discipline that seeks to understand basic brain function as well as cure these disorders. Experimental neuroscience started arguably $\sim 50$ years ago, and nowadays a considerable number of important therapeutic approaches can be traced back to findings from animal experiments. For instance, chip-based retina implants, which enable the blind to have a basic vision, or cochlear implants for the deaf are based on scientific experiments with chickens, rabbits, cats, pigs, and non-human primates. Deep brain stimulation combines neurosurgical and electrophysiological approaches that were developed primarily in non-human primate models (Wichmann et al., 2018). It is now used successfully in patients with Parkinson's disease and associated movement disorders.

Despite these successes, we generally know little about brain diseases. This is painfully illustrated by the fact that many diseases of the brain are currently not classified by their cause but by specific symptom clusters. For instance, the current classification system places patients suffering from a chronic major depression with severe anhedonia and weight loss 
A

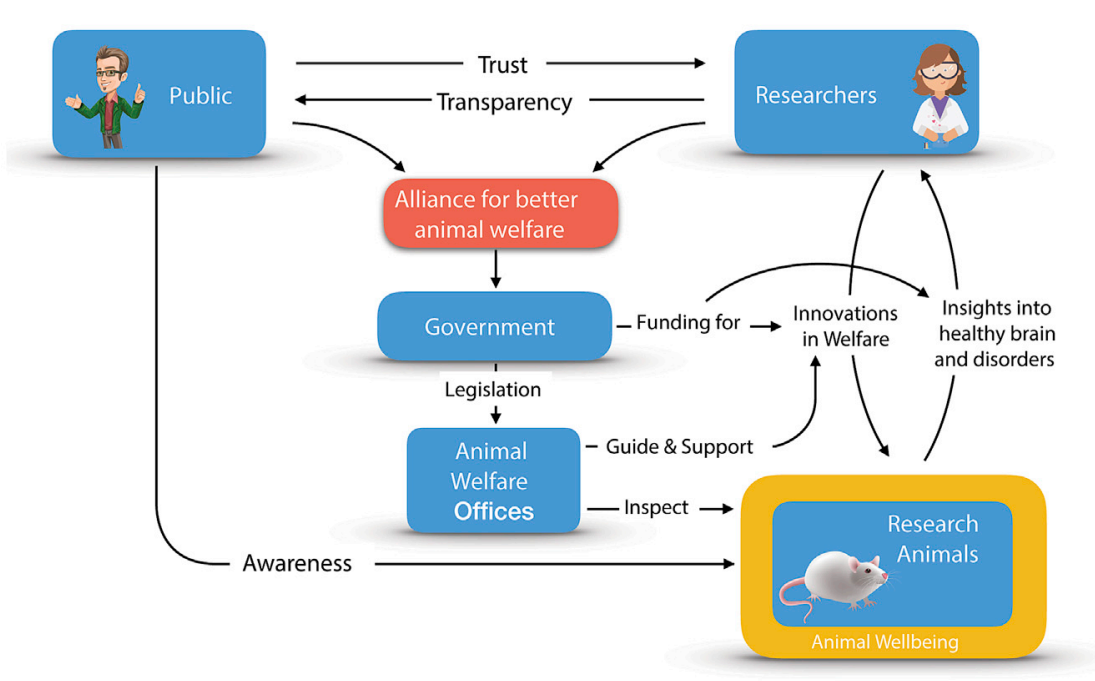

B

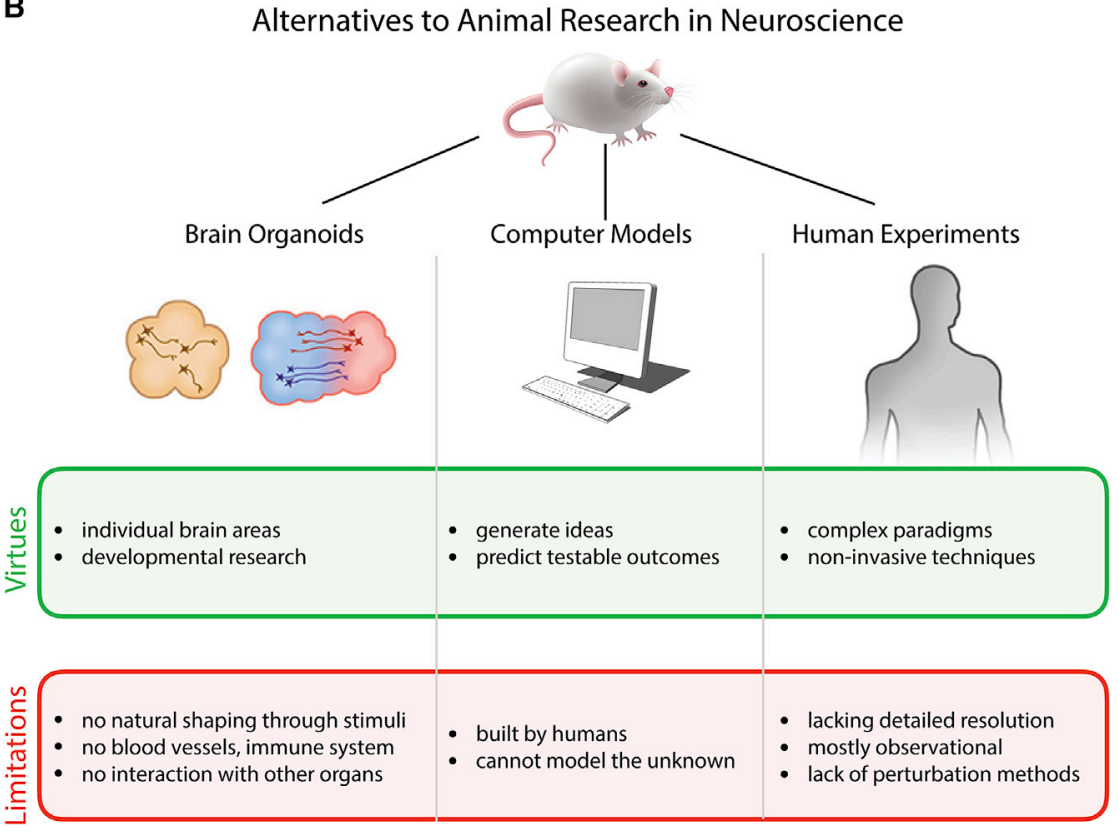

Figure 1. The public, regulators, and researchers share the goal of improving animal welfare (A) Research in live animals is central to gaining deeper insights into the functioning of the healthy and diseased brain. A high standard of animal welfare is central to arriving at reliable results, and researchers are therefore often introducing innovations in animal welfare. These improvements are to the benefit of the animals and are supported by regulators and the public. However, insufficient funding can slow down or prevent innovations in animal welfare, and we therefore advocate an alliance between the public and researchers to convince the funding agencies to add funding to existing programs that can be used specifically for animal welfare innovations.

(B) Shown are some alternatives to animal research and the virtues as well as limitations. These alternatives complement rather than replace animal research in neuroscience.

within the same classification as those showing shorter and lighter depressive episodes combined with sleep and weight gain problems. The reason is that we lack sufficient understanding of the core mechanisms underlying depression and, thereby, who will respond to what intervention. As a result, instead of tackling the underlying disease mechanism, we currently treat many of the symptoms based on serendipity, or according to "try-and-change" protocols.

The field of neuroscience also tries to obtain basic understanding of how the brain, consisting of billions of neurons (Azevedo et al., 2009), can mediate essential processes like sleep, eating, social and sexual behavior, movement, emotion, thinking, planning, imagination, and memory. The brain has bi-directional communication with the gut, heart, and lungs, receives information from sensory cells distributed all over the body, and has multiple interactions with the immune and endocrine systems. It is thus more complex and all-encompassing than any other organ in our body. Also, the brain is highly individual and, because its regenerative potential is limited, also irreplaceable. While the brain is so fundamental in health and disease, at the same time it is the organ we know the least about. Without properly understanding the healthy brain, it is impossible to understand diseases that affect the brain, let alone to develop appropriate therapeutics. Animal research is indispensable for this understanding as it generates fundamental knowledge about the function and structure of the healthy brain in order to develop new hypotheses for human and veterinary research, validate, or back-translate, human findings, and develop therapeutic approaches for brain diseases.

One example of how little we understand the brain is seen in memory research. It was generally believed that memories are stored by strengthening the connections between neurons, i.e., the brain cells that communicate via the synapse. Yet, new technologies allowing resolution at the cellular level have revealed that many more elements in the brain contribute to memory. Astrocytes (Perea et al., 2014), glial cells that support metabolic functions in the brain; oligodendrocytes, cells that create the myelin-sheath that isolates neuronal connections; and the extracellular matrix, proteins surrounding all these cells, have all been shown to be critical for specific aspects of memory. These new findings have considerable implications for our understanding of conditions such as dementia and highlight that much of what we thought we knew may actually only 
reflect a very small part of the whole picture.

\section{Alternatives to animal research in neuroscience are important}

In order to advance the understanding of the maturing brain, we are making substantial progress in developing brain organoids, which are cultured miniature brain parts derived from human induced pluripotent stem cells (hIPSCs) that offer unprecedented new ways to study aspects of human brain development. These brain organoids can model human brain disease and help screen for drug effects at the molecular and cellular level. However, a brain organoid is by no means a complete brain and presents several limitations: (1) they model only part of the brain, lacking any instructive neurotrophic signals from other brain areas; (2) they miss relevant components, such as functional blood vessels and an immune system; and (3) they lack bi-directional interactions with other organs in the body. As such, organoids are promising for specific goals such as developmental research but not yet applicable for several other research goals. Indeed, a brain organoid is deprived of sensory input from the body and hence lacks the ability to interact with the outer world. This is crucial because the brain's connectivity and function is shaped, in large part, in the context of sensory experience. Furthermore, understanding brain function is almost always tied to behavior and cognition. Organoids obviously cannot "behave." Therefore, while organoids are extremely promising for specific goals at the cellular or molecular level in neuroscience research, the potential to use these model systems as an alternative to animal research is limited in neuroscience. In fact, organoids are a complementary approach that are most often combined with research on the whole animal, for instance, to validate in vitro findings in vivo. For all of these reasons, we argue that brain organoids complement animal research and can help to reduce animal use but cannot be used to completely replace animal research, particularly not behavioral neuroscience research.

Computer models are also discussed as an alternative. However, these are built by humans using the current available knowl- edge. Knowledge is by definition incremental and without limits, yet a computer model can never be created based on processes we do not know exist. Instead, computer models are the most effective in neuroscience research when built and used in close collaboration with animal research (Churchland and Sejnowski 2016). Computer models can be used to generate ideas and predict outcomes that then can be tested in a live neuronal system (Richards et al., 2019). Therefore, they can aid animal research and potentially lead to a reduction in the number of animals used but never fully replace animals in science.

Another alternative for animal research is to shift to research where humans themselves participate as research subjects. This is common practice in neuroscience, whereby the brain is studied via non-invasive techniques such as neuroimaging (magnetic resonance imaging [MRI]) and electroencephalography. However, while these techniques have significantly advanced the understanding of brain structure and function in health and disease, the readouts are often only indirect and lack the resolution to assess more molecular mechanistic aspects of brain structure and function. Due to evident practical and ethical constraints, experimental interventions in humans are limited and almost all research in humans remains observational.

Overall, in the field of neuroscience, several in vitro approaches, computer models, and human research are applied to help answer specific sub-questions and together with animal research complement each other, but many research questions that focus on unravelling the fundamental mechanisms will still require experimentation in intact live animals (Figure 1B).

Various animal species are used in neuroscience

The most commonly used animal species in neuroscience-related laboratory research are rodents, i.e., mice and rats. Other species used in neuroscience research include zebrafish, C. elegans, fruit flies, hamsters, guinea pigs, sheep, tree shrews, and non-human primates. Each animal species has specific traits that make them the most suitable model system for a specific basic or disease- related question. While we share the ethical worries associated with studying non-human primates, it has to be recognized that much of what we know today about neural activity in the cortex during complex behavior, visual processes, and higher cognitive functions has been discovered in non-human primate model studies (Goldberg, 2019). Specifically, the prefrontal cortex is more alike between non-human primates and humans than between rodents and humans (Passingham et al., 2013). Non-human primates thus provide the highest level of face validity, as their brains are organized in the most similar way to the human brain.

Animal research in neuroscience is bound to strict regulations

As in all animal research, neuroscientific animal research is bound by strict regulations in most countries around the world (Mitchell et al., 2021). While each country will have its own implementation of ethical review and regulation, many agree that vertebrate species should be protected. For example, many European countries require two types of ethical permission: an initial general project license covering a longer period (up to 5 years) and, subsequently, approval of the actual experiment by the local review bodies. In other countries, only one approval step is required. In each case, the animal experiments must be demonstrably scientifically sound and must have scientific or societal relevance. The 3Rs (replacement, reduction, and refinement) are key in this evaluation process: it is not permitted to conduct animal research when alternatives are available, and the research must use the most refined procedures and the least number of animals (without compromising scientific quality).

The ethical regulations in place across Europe and in many other countries are strict and work well to protect and improve animal welfare. In some countries, however, these regulations have become overburdened by bureaucracy (Genzel et al., 2020). For example, the process of receiving the first ethical approval should, on average, take approximately 6 months. However, in reality, the total process often takes much longer, e.g., in Germany, $80 \%$ of applications are not processed in time so that paperwork and approvals often take 
longer than the duration of the proposed experiment. If one has to deviate from the ethical permit and work protocol, an amendment of the ethical permit is required, meaning that even making changes to use fewer animals or perform fewer measurements requires express permission. This bureaucracy increases impediments to discovery research.

\section{Basic research, understanding} diseases, and seeking treatments When considering neuroscience research using animals, one can make a distinction between different types of scientific research. There is (1) basic research, which aims at understanding how fundamental processes work in healthy animals, including humans. Then, there is (2) basic preclinical research, which aims at understanding disease by characterizing the symptoms, delineating the affected molecular circuits or systems and investigating the underlying causes and mechanisms. Finally, there is (3) applied pre-clinical research, which is aimed at the identification of treatments and testing their efficacy.

It is important to distinguish among these types of research because each is essential yet comes with a different approach. When thinking of animal research, most people have applied preclinical research in mind. The study design that is typically thought of is the testing of compounds in alleviating diseases. As a result, legislation is geared toward this type of research. In this type of research, the hypotheses are clear, and it is possible to conduct power analyses to estimate the group sizes needed for the experiments. However, in basic and basic preclinical research, researchers are more frequently dealing with unknown processes that remain to be discovered. To allow for new discoveries to be made, which are necessary to advance basic knowledge, more flexibility and research-type-specific regulations are needed.

\section{Unexpected discoveries cannot be planned}

In science, one cannot plan what is not known. Many important scientific discoveries relied on serendipity. One example is the discovery of grid cells that led to the Nobel Prize in Physiology and Medicine in 2014. When the key investigators,
May-Britt and Edvard Moser started their investigations, they knew about place cells from the work by John O'Keefe (place cells fire in accordance to where an animal is in space) in a specific part of the brain, the hippocampus. They aimed at understanding how the brain can create this signal and therefore decided to look into a brain area that feeds inputs into the hippocampus, the entorhinal cortex, without having a clear idea of what they would find. Their basic research led them to find grid cells: cells that fire at a regular distance in spatial environments and therefore allow the brain to create the place cell signal. The discovery of both place cells and grid cells was a breakthrough in science and was of the utmost importance for our understanding of how the brain enables spatial and temporal orientation and navigation, which is of high relevance for the understanding of the brain as well as brain disorders characterized by hippocampal neuropathology.

With the current trend to overinterpret regulations, this type of discovery (basic) research is getting harder to do. In several European countries, the rules that have been implemented leave very little room for experimental flexibility and require substantial detail at the initial ethical approval level. For the hard research questions, including fundamental unknowns, it is impossible to lay out a detailed 5-year experimental plan that will not be amended or changed. Basic research is based on finding something new and following up on this new finding, often within the ongoing experiment, with progress from one step to the next often within weeks or months. National ethics protocols should be more tailor made, taking into account the type of research (basic, basic preclinical, preclinical applied) at hand. In addition, a clearer and more expedient process for writing and reviewing protocols for particular types of experiments, especially those including yet unknown factors, is needed within the legislation for all countries.

\section{Conclusion}

To keep neuroscience research viable and continue making progress, we think that policymakers need to listen more carefully to animal researchers. Animal rights activists often imply that animal researchers do not care about animal welfare. This could not be more wrong: animal researchers care a great deal about their animals (Mitchell et al., 2021). Researchers aim to collect sound experimental data and do not want stressed animals unless they are specifically investigating stress, which is done under very controlled conditions. When improvements are made in animal welfare, they are often introduced by the researchers themselves, in the context of limited funding (Figure 1A). Therefore, we advocate that the public and the scientific community should come together as partners for an alliance for better animal welfare, which convinces governments and regulators to provide funding specifically for refinements in experimentation that benefit animal welfare.

We are concerned that extremely strict regulation can sometimes harm rather than benefit the animals. In some circumstances, the delays in obtaining permission to follow up on an unanticipated observation in experiments mean that the animals have grown too old and that the entire experiment must be replicated in new animals. Or we conduct suboptimal experiments because it is not possible to deviate from what has been approved. We are concerned that a new trend toward further regulation of animal research could greatly increase the administrative burden for researchers and will hinder progress in the field. Further, when the atmosphere in which neuroscientists have to work becomes unbearable, researchers feel their only option is to emigrate to other more welcoming countries.

In summary, there is currently no foreseeable end to the need for animal research in neuroscience. While we hope that one day in the future our knowledge will become so comprehensive as to no longer require animal research, we must face the fact that we are far from that scenario. Neuroscience is at an exciting stage, where fundamental progress is being made at an ever-increasing pace. Single-cell transcriptomics revealed that there are thousands, if not tens of thousands, of cell types in the brain. The neuroscience community has recently developed tools to causally interfere with small cell populations using optogenetic or pharmacogenetic approaches that will 
allow us to finally go beyond just observing the brain and delineate detailed mechanisms. Some of these techniques even have potential as therapeutics. The development of new technologies in neuroscience that has just recently allowed us to have a streak of discoveries could be stopped in its tracks if we compromise animal research now.

\section{ACKNOWLEDGMENTS}

We would like to thank Loren Frank, UCSF, USA; Sheena Josselyn, Hospital for Sick Children, University of Toronto, Canada; Shantanu Jadhav, Brandeis University, USA; the European Animal Research Association (EARA); the Federation of European Neuroscience Societies Committee on Animals in Research (CARE); the Swiss Society for Neuroscience; the Society for Neuroscience Committee on Animals in Research (CAR); and Stichting Informatie Dierproeven (the Dutch foundation for public information on animal testing: SID) for input on and support for this article.

\section{REFERENCES}

Azevedo, F.A., Carvalho, L.R., Grinberg, L.T. Farfel, J.M., Ferretti, R.E., Leite, R.E., Jacob Filho, W., Lent, R., and Herculano-Houzel, S. (2009). Equal numbers of neuronal and nonneuronal cells make the human brain an isometrically scaled-up primate brain. J. Comp. Neurol. 513, 532-541.

Churchland, P.S., and Sejnowski, T.J. (2016). Blending computational and experimental neuroscience. Nat. Rev. Neurosci. 17, 667-668.

Genzel, L., Adan, R., Berns, A., van den Beucken, J.J.J.P., Blokland, A., Boddeke, E.H.W.G.M., Bogers, W.M., Bontrop, R., Bulthuis, R. Bousema, T., et al. (2020). How the COVID-19 pandemic highlights the necessity of animal research. Curr. Biol. 30, R1014-R1018.

Goldberg, M.E. (2019). The neurology clinic needs monkey research. Proc. Natl. Acad. Sci. USA 116 , 26255-26258.

Mitchell, A.S., Hartig, R., Basso, M.A., Jarrett, W. Kastner, S., and Poirier, C. (2021). International primate neuroscience research regulation, public engagement and transparency opportunities. Neuroimage 229, 117700.

Passingham, R.E., Rowe, J.B., and Sakai, K. (2013). Has brain imaging discovered anything new about how the brain works? Neuroimage 66 142-150.

Perea, G., Yang, A., Boyden, E.S., and Sur, M. (2014). Optogenetic astrocyte activation modulates response selectivity of visual cortex neurons in vivo. Nat. Commun. 5, 3262.

Raggi, A., and Leonardi, M. (2020). Burden of brain disorders in Europe in 2017 and comparison with
other non-communicable disease groups. J. Neurol. Neurosurg. Psychiatry 91, 104-105.

Richards, B.A., Lillicrap, T.P., Beaudoin, P., Bengio, Y., Bogacz, R., Christensen, A., Clopath C., Costa, R.P., de Berker, A., Ganguli, S., et al. (2019). A deep learning framework for neuroscience. Nat. Neurosci. 22, 1761-1770.

Wichmann, T., Bergman, H., and DeLong, M.R. (2018). Basal ganglia, movement disorders and deep brain stimulation: advances made through non-human primate research. J. Neural Transm. (Vienna) 125, 419-430. 